\title{
Stability of auditory evoked potentials during simultaneous visual stimulation
}

\author{
LYNN C. OATMAN \\ U.S. Army Human Engineering Laboratory \\ Aberdeen Proving Ground, Maryland
}

\begin{abstract}
During sensory interaction, an accessory stimulus in a second modality can have a facilitating or inhibiting effect upon some measurable response to a stimulus applied to a primary modality. This study examined whether increased intensity of an accessory light stimulus facilitates or inhibits auditory evoked potentials to click stimuli. Click-evoked potentials were recorded from the round window (cochlear microphonic and auditory nerve), cochlear nucleus, and auditory cortex of unanesthetized cats, and measured before, during, and after simultaneous accessory light stimulation of different intensities. At all electrode sites, the mean peak-to-peak amplitudes of clickevoked potentials were not significantly different during simultaneous visual and auditory stimulation when compared with the pretest and posttest control periods of auditory stimulation. In addition, at all sites, the amplitude of the click-evoked potentials remained constant as accessory light stimulation was increased in intensity. The results suggest that sensory interaction effects do not occur when both the auditory and visual stimuli are irrelevant. If such effects do occur, they are so weak as to be undemonstrable for a wide range of visual stimulus intensities.
\end{abstract}

During sensory interaction, a stimulus presented to one sensory modality has either a facilitating or an inhibiting effect upon some measurable response to a stimulus applied to a second sensory modality. The behavioral studies examining sensory interactions have been contradictory, particularly the research examining the effects of accessory visual stimulation upon the absolute threshold of hearing sensitivity. Research has shown the simultaneous accessory visual stimulation as facilitating (Child \& Wendt, 1938; Gregg \& Brogden, 1952; Klayman, 1973; O'Hare, 1956; Seif \& Howard, 1975; Thompson, Voss, \& Brogden, 1958; Watkins, 1966), as inhibiting (Jacobson, 1911; Markov, 1960), or as having no effect (Bothe \& Marks, 1970; Gulick \& Smith, 1959; Howarth \& Treisman, 1958; Karlovich, 1969; Sheridan, Cimbalo, Sills, \& Alluisi, 1966) upon sensitivity in the primary auditory modality.

It is difficult to reconcile these diverging results, although one factor that may have contributed to the contradictory nature of the data on sensory interaction is the intensity of the accessory stimulus acting upon the primary modality. Intensity has been suggested as an important variable in sensory interaction (Gulick \& Smith, 1959; Seif \& Howard, 1975; Shigehisa \& Symons, 1973a). The results of these studies have not only indicated that the

In conducting the research described herein, the investigator adhered to the Guide for Laboratory Animal Facilitaties for Laboratory Animal Resources, National Academy of Sciences, National Research Council, Washington, D.C. This paper is part of the public domain and may be reproduced in full or in part for any purpose of the United States Government. The author gratefully acknowledges the assistance of Donna $\mathbf{M}$. Kramer in reducing the data. The author's mailing address is: Behavioral Research Directorate, U.S. Army Human Engineering Laboratory, Aberdeen Proving Ground, MD 21005. accessory effect can be either facilitating or inhibiting, but also have suggested that the direction of the effect may be influenced by the intensity of the accessory stimulus (Shigehisa \& Symons, 1973a). Shigehisa and Symons (1973a) reported that an increase in auditory sensitivity was typically found under weak accessory stimuli, and a decrease under strong stimuli. However, previous studies have not explored the possibility that the intensity of the accessory stimulus could exert a direct effect on the evoked responses in the primary modality. Thus, the relationship between the intensity of the accessory stimulus and the sensitivity in the primary modality remains to be quantified and the point of transition between facilitation and inhibition determined.

The purpose of this experiment was to determine whether increased intensity of an accessory light stimulus facilitated or inhibited auditory evoked potentials to click stimuli. Although most studies of sensory interaction have measured the psychophysical threshold in the classic sense, the present study employed amplitude measures of the auditory evoked potentials. The contradictory results obtained in the earlier studies could perhaps be accounted for by the unreliability of the sensory response measures used, which were not always sensitive enough to detect or measure the magnitudes of the accessory effects, particularly in studies in which the intensity of the accessory stimulus was not controlled (Shigehisa \& Symons, 1973b). A more reliable measure could perhaps be provided by an examination of the auditory evoked potentials for changes in amplitude during simultaneous accessory visual stimulation.

The study was designed to determine if click-evoked potentials recorded along the auditory pathway were suppressed in amplitude while an animal, whose middle-ear 
muscles had been severed, was simultaneously receiving accessory visual stimulation. In previous studies, Oatman $(1971,1976)$ had observed that auditory evoked potentials at the receptor level and the cortical level were suppressed in amplitude while an animal whose middle-ear muscles had been severed was attending to visual stimulation. Oatman suggested that during attention to visual stimulation a central inhibitory mechanism suppressed irrelevant auditory stimuli through the action of the olivocochlear bundle. In those studies, perhaps the observed suppression of auditory evoked responses was actually the result of sensory interaction, rather than the result of attention mechanisms. To examine this possibility, the experimental paradigm used in this experiment was the same as that used in the attention studies (Oatman, 1971, 1976, 1982; Glenn \& Oatman, 1977; Oatman \& Anderson, 1977, 1980), although no attempt was made to alter the attentive state of the subjects through conditioning procedures. In brief, auditory evoked potential amplitudes were determined before, during, and after simultaneous accessory light stimulation at seven levels of intensity.

\section{Preparation of Subjects}

\section{METHOD}

Electrodes were placed on the round window and bilaterally in the cochlear nucleus and auditory cortex of six female cats, each weighing approximately $2.5 \mathrm{~kg}$, under sodium pentobarbital anesthesia $(0.5 \mathrm{cc} / \mathrm{kg}$ at a concentration of $65 \mathrm{mg} / \mathrm{cc}$ ). The cochlear nucleus electrodes were stereotaxically implanted through small holes bored in the skull according to coordinates in the stereotaxic atlas of Snider and Niemer (1961). The concentric bipolar electrodes were made of $0.2-\mathrm{mm}$ stainless steel wire and inserted into 0.5-mm stainless steel tubing (Rhodes Model NEX100 ). Both the wire and the tubing were coated with an Epoxylite insulator up to $0.5 \mathrm{~mm}$ from the tip; the tips were $1.0 \mathrm{~mm}$ apart. The cortical electrodes were flattened monopolar silver-ball electrodes stereotaxically implanted on the dura over the auditory cortex (AI). The round window electrode was a 0.2 -mm ball-tipped stainless steel wire in polyethylene tubing. At the time of the round-window implantation, the tendons of the stapedius and tensor tympani muscles were cut. The indifferent electrode was a stainless steel screw placed over the frontal sinus; another stainless steel screw, placed at the posterior part of the skull, was used as an internal ground for the animal. A detailed explanation of the surgical preparation used in this experiment can be found elsewhere (Oatman, 1971, 1976).

\section{Histology}

At the end of the experiment, the cats were given a lethal overdose of sodium pentobarbital administered intravenously. Electrolytic lesions were produced at the recording sites of each concentric electrode. The lesion current was $1 \mathrm{~mA}$ for $15 \mathrm{sec}$. The brains were removed and placed in Formalin and potassium ferrocyanide. All placements were verified histologically using unstained, frozen sections (Siegel, 1968). For all six cats, the histology slides confirmed that the electrodes were placed in the dorsal cochlear nucleus. Examination of the brains confirmed that the auditory electrodes were placed in the middle ectosylvian gyrus (AI). Middle ears were examined with a Bausch and Lomb Stereozoom seven dissecting microscope to determine that the middle-ear muscle tendons had been completely severed.

\section{Visual and Acoustic Stimulation}

The visual stimuli consisted of light presented to one eye of the cat. The light stimuli were presented for a duration of $8 \mathrm{sec}$ and at a rate of 1 every $25 \mathrm{sec}$ at each of seven intensities ranging from 3 to $30 \mathrm{fc}$. Luminance measurements were made on the incandescent lamp with a Pritchard spectrophotometer (Model 1970-PR). The light stimuli were generated by an 8-sec square-wave pulse (Tektronix Type 26G3), which activated a small incandescent lamp (Subminiature lens-end lite, Type CM 20-3). The lamp was placed approximately $.635 \mathrm{~cm}$ in front of the cat's eye. The lamp was not fastened to the eye but was held firmly in place by a bracket attached to the electrode plug.

Auditory clicks were presented continuously at a rate of $1 / \mathrm{sec}$ during the presentation of the light stimuli, but they were not synchronized with the onset of the light stimuli (Figure 1). The auditory clicks were generated by a $90-\mu \mathrm{sec}$ square-wave pulse (Tektronix 26G3). The puises were led through a high-pass filter (Allison Laboratories Model 2-B, $4800 \mathrm{~Hz}$ ), and through a decade attenuator (General Radio Type GR-1450) and a power amplifier (Crown Model D-150A) to a driver (University Model ID-60). The clicks were presented at an intensity of $85 \mathrm{~dB}$ SPL (re .0002 microbar) through a sound-tube system which terminated at the entrance to the cat's external meatus. The sound tube was not fastened to the pinna but was held firmly in place by a bracket attached to the electrode plug. This intensity $(85 \mathrm{~dB})$ was selected because the cochlear microphonics were in the linear portion of its input-ouput function. Sound pressures were calibrated with a $.635-\mathrm{cm}$ condenser microphone (Bruel and Kjaer Type 4135) and placed perpendicularly to and just in front of the end of the sound tube. Movements of the sound tube to different positions within the test cubicle did not change the output voltage from the microphone.

\section{Data Collection and Procedure}

Simultaneous recordings were obtained from the round window (cochlear microphonics and auditory nerve responses), the cochlear nucleus, and the auditory cortex in response to click stimuli. Recordings were obtained from unrestrained animais via a Microdot shielded cable connected to an electroencephalograph (Grass Model 7) placed outside the sound-attenuating cubicle. At the same time, the click-evoked potentials were recorded on a 14-channel FM tape recorder (Sangamo 4700), from which they were led into a signal averager (Nicolet Med 80) and written on an X-Y plotter (Hewlett-Packard 7035B).

Four weeks after surgery, the cats were placed into the soundattenuating test cubicle and the electrodes were checked. Figure 2 shows an example of the click-evoked responses from the round window (CM and N1-N2), cochlear nucleus, and auditory cortex, and indicates how the peak-to-peak measurement was made for each electrode placement. Peak-to-peak amplitudes of the averaged evoked responses were measured by computer. Evoked potentials influenced by bodily movement as observed in the EEG were discarded from the data.

After the electrodes were checked, the data were collected in recording sessions that consisted of three different periods: (1) a pretest control in which the cat was awake, relaxed, and receiving only click stimuli; (2) an experimental period in which the cat was receiving visual and auditory stimuli simultaneously; and (3) a posttest control period similar to the pretest control period. The click-evoked responses, averaged during the experimental period, included only those evoked potentials

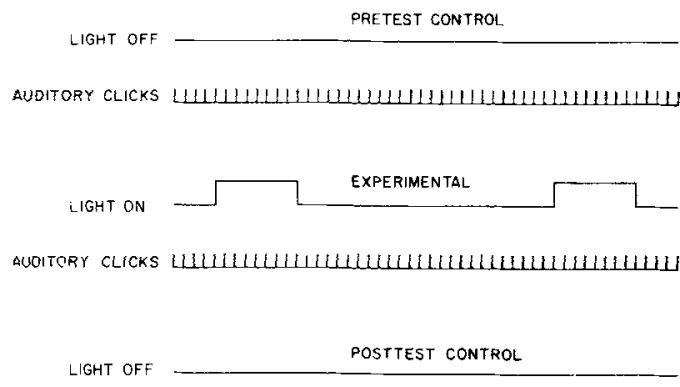

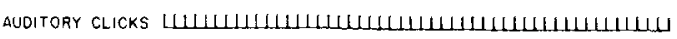

$$
\underset{0}{W \amalg L \amalg \amalg}
$$

Figure 1. Temporal relationships between the auditory and visual stimuli for experimental and control conditions. 


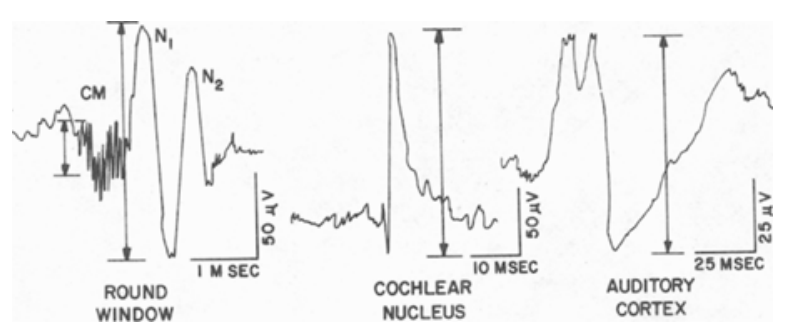

Figure 2. Averaged click-evoked responses recorded from the round window, cochlear nucleus, and auditory cortex. The round window wave form shows cochlear microphonics (CM) and auditory nerve (N1, N2) responses to a single click. The peak-to-peak conventions used to quantify the evoked potentials are noted.

presented during the 8-sec light-on period (Figure 1). The three different recording sessions, pretest control, experimental, and posttest control, were conducted on the same day. Prior to data collection, 2 drops of Mydriacyl 1\% ophthalmic solution were applied to each eye of the cat in order to dilate the pupils. Evoked potentials to clicks $(85 \mathrm{~dB})$ for each of the three different recording sessions were then collected at each of seven different light intensities (one intensity per day) in an ascending order of presentation from 3 to $30 \mathrm{fc}$.

\section{RESULTS}

The data consist of averages of 64 click-evoked potentials from three electrode locations: round window (cochlear microphonic and auditory nerve), cochlear nucleus, and auditory cortex. The data plotted in Figures 3 and 4 are averages obtained from 512 measurements from each electrode placement recorded on each of six cats. These figures show the average peak-to-peak amplitudes of the auditory responses plotted as a function of visual intensity for each of the three recording sessions: pretest control period (cat received only auditory click stimuli), experimental period (cat received auditory clicks and visual light stimuli simultaneously), and posttest control period (cat received only auditory click stimuli).

Figure 3 shows the mean peak-to-peak amplitudes of auditory cortex click-evoked potentials as a function of recording session and increased visual light intensity. An analysis of variance (Butler, Kamlet, \& Monty, 1969) indicated no significant differences between the recording sessions (pretest control, experimental, and posttest control) $[\mathrm{F}(2,100)=2.54]$, and no significant differences as a function of increased light intensity $[\mathrm{F}(6,100)=$ 2.06].

The mean peak-to-peak amplitudes of the cochlear nucleus, auditory nerve, and cochlear microphonic responses appear in Figure 4 as a function of recording session and increased light intensity. The figure shows that the clickevoked potentials changed very little with recording session and increased light intensity. An analysis of variance indicated no significant differences across the recording sessions for the auditory nerve $[\mathrm{F}(2,39)=1.71]$, the cochlear nucleus $(F<1)$, and the cochlear microphonics $[F(2,39)=1.84]$. Increased light intensity had no significant effects on the auditory evoked potentials of the auditory nerve $[\mathrm{F}(6,39)=1.62]$, the cochlear nucleus
$[F(6,100)=1.02]$, and the cochlear microphonics $[F(6,39)=1.90]$. The figure also shows that the largest mean amplitudes were obtained from the auditory nerve and the smallest mean amplitudes from the cochlear microphonics; the cochlear nucleus mean amplitudes were in between. These mean-amplitude differences reflect differences in the electrode placements and electrical sources.

\section{DISCUSSION}

The experimental results demonstrated that accessory visual stimulation had no systematic effect on the clickevoked responses of the peripheral (CM, N1, or $\mathrm{CN}$ responses) or central (AC responses) auditory systems. For the parameters used in this study, it seems unlikely that click-evoked potentials recorded from the auditory pathways are either facilitated or inhibited during increased intensity of an accessory visual stimulus. These negative findings are consistent with the previous reports of sensory interaction (Bothe \& Mark, 1970; Gulick \& Smith, 1959; Howarth \& Treisman, 1958; Karlovich, 1969; Karlovich \& Moore, 1971), which also were un-

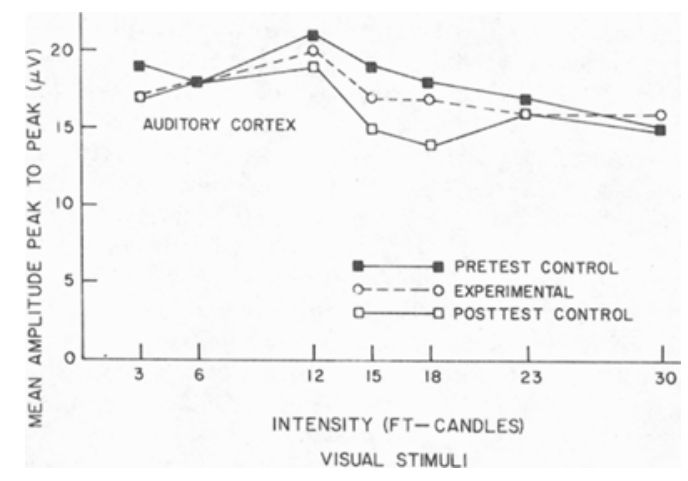

Figure 3. The mean peak-to-peak amplitude of auditory cortex evoked potentials in microvolts as a function of the intensity of simultaneous light stimuli (experimental) and no light stimuli (pre- and posttest controls).

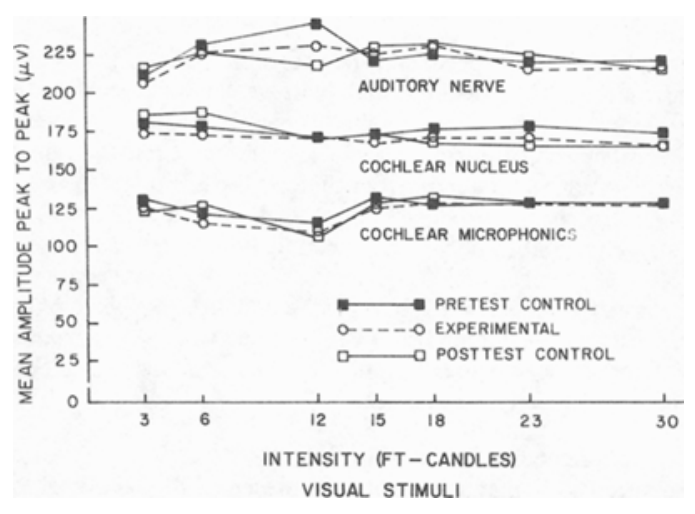

Figure 4. The mean peak-to-peak amplitude of auditory nerve, cochlear nucleus, and cochlear microphonics as a function of the intensity of simultaneous light stimuli (experimental) and no light stimuli (pre- and posttest controls). 
able to demonstrate a sensory interaction between the visual and auditory systems. An evaluation of these experiments (Gulick \& Smith, 1959; Karlovich, 1969; Karlovich \& Moore, 1971; Sheridan et al., 1966) revealed that subjects were given no instructions regarding the processing of accessory visual stimuli. In fact, emphasis was placed on the primary auditory task, and all other accessory stimuli were identified as irrelevant to the subject. The lack of effect observed in these studies could be due to the fact that no processing of the irrelevant accessory visual stimuli was required. Likewise, the source of failure to demonstrate sensory interaction in the present study could be the lack of relevance of the accessory visual stimuli and/or auditory click stimuli. Apparently, when both the auditory and visual stimuli are irrelevant, as in the present study, unattended stimuli are not required to be processed beyond the identification of their simple physical characteristics (Hillyard, Picton, \& Regan, 1978).

The approach taken in this experiment was to use the amplitude measures of the auditory-evoked potentials, as an index of sensory-interaction effects, rather than measures of the psychophysical threshold of hearing sensitivity. It might be argued that the amplitude of the auditory evoked potential was simply insensitive as a sensory response measure and, therefore, not sufficient to detect measureable magnitudes of the accessory stimulus effects. This argument seems unlikely since the evoked potential thresholds have been shown to be highly correlated to the behavioral thresholds that most studies use to measure sensory-interaction effects. Sensory evoked-potential components have thresholds that are near or slightly above the behavioral threshold (Hillyard, Squires, Bauer, \& Lindsay, 1971; Hillyard et al., 1978; Paul \& Sutton, 1972).

Although the arousal and warning properties of the accessory visual stimuli are of traditional concern, they are not of concern in the present experiment, since the data presented indicate that, within the limits studied, auditoryevoked potentials are either not differentially affected or only very weakly affected, and thus difficult to demonstrate by accessory visual stimulation or increased intensity of the accessory visual stimulation. Although it is possible that extremely intense light would be arousing and therefore might affect auditory-evoked potential amplitudes, this effect might better be viewed as the result of distraction rather than sensory interaction (Gulick \& Smith, 1959).

In contrast to the data reported here, previous studies (Child \& Wendt, 1938; Gregg \& Brogden, 1952; Klayman, 1973; Seif \& Howard, 1975; Shigehisa \& Symons, 1973a; Thompson et al., 1958) have demonstrated that sensory interaction does occur between the auditory and visual modalities, and have suggested that the intensity relationship of the accessory stimuli acting upon the primary modality is involved in demonstrations of sensory interaction (Shigehisa \& Symons, 1973b). Perhaps these conflicting results in sensory-interaction experiments are due to attentional mechanisms, since facilitation might be expected if the subject's attention was focused on the primary stimulus and inhibition might be expected if the subject's attention was centered on the accessory stimulus. In the previous studies demonstrating sensory interactions, it seems quite likely that attention was inadvertently directed to the second modality by requiring the subjects to make a behavioral response to the accessory visual stimuli. However, it is unclear if subjects only occasionally switch their attention to the irrelevant accessory modality or if they make a continuous analysis of its stimuli. Since several investigators (Bothe \& Marks, 1970; Gulick \& Smith, 1959; Howarth \& Treisman, 1958; Taylor, 1974) have suggested that attentional factors clearly play a role in demonstrations of sensory interactions, it may be supposed that sensory-interaction effects are due to the allocation of attention to the primary and secondary modalities, with the primary modality typically being more heavily attended than the secondary modality. One advantage of the evoked-potential measures is that it is possible to assess allocations of attention to relevant and irrelevant classes of stimuli in the absence of overt motor responses (Hansen \& Hillyard, 1983; Hillyard et al., 1978). Thus, the lack of sensory-interaction effects seen in the present study could be due to the fact that the subjects were not required to allocate attention to either the primary or the secondary modality.

One of the major concerns of the present study was to determine whether the suppression of auditory-evoked responses observed in previous attention studies (Oatman, $1971,1976,1982$ ) was actually the result of auditoryvisual interaction. The present study examined this possibility by using the same experimental procedures that had been used in the attention studies, except that no attempt was made to alter the attentive state of the subjects through conditioning procedures. The middle light intensity $(15 \mathrm{fc})$ in this study was identical in intensity to the visual discrimination task used in the previous attention studies. Three intensities were selected above and below this value to examine the intensity relationship of visual stimulation per se on auditory evoked potentials. A major effort was made to keep the conditions equivalent for the attention and nonattention experiments, except for the alteration of the attentive state through conditioning procedures in the attention studies and the changes in intensity of the accessory visual stimulation in the nonattention studies. The present experiment showed no changes in the amplitude of auditory evoked potentials with increased light intensity stimulation. In fact, no evidence of auditoryvisual interaction was obtained under any of the experimental conditions used. Based on these results, it can be concluded that suppression of auditory evoked potentials demonstrated in previous attention studies did not result from sensory interaction.

\section{REFERENCES}

Bothe, G. G., \& Marks, L. E. (1970). Absolute sensitivity to white noise under auxiliary visual stimulation. Perception \& Psychophysics, 8, 176-177. 
Butler, D. H., Kamlet, A. S., \& Monty, R. A. (1969). A multipurpose analysis of variance FORTRAN IV computer program. Psychonomic Monograph Supplement, 2(16, Whole No. 32).

ChILD, I. L., \& WENDT, G. R. (1938). The temporal course of the influence of visual stimulation upon the auditory threshold. Journal of Experimental Psychology, 23, 109-127.

GlenN, J. F., \& OATMAN, L. C. (1977). Effects of visual attention on the latency of auditory evoked potentials. Experimental Neurology, 57, 34-40.

GreGG, L. W., \& Brogden, W. J. (1952). The effect of simultaneous visual stimulation on absolute and auditory sensitivity. Journal of Experimental Psychology, 43, 179-186.

Gulick, W. L., \& SMITH, F. L. (1959). The effect of intensity of visual stimulation upon auditory acuity. Psychological Record, 9, 29-32.

Hansen, J. C., \& HillyaRd, S. A. (1983). Selective attention to multidimensional auditory stimuli. Journal of Experimental Psychology: Human Perception and Performance, 9, 1-19.

Hillyard, S. A., Picton, T. W., \& Regan, D. M. (1978). Sensation, perception, and attention: Analysis using ERPs. In E. Callaway, P. Tueting, \& S. Koslow (Eds.), Event-related brain potentials in man (pp. 223-321). New York: Academic Press.

Hillyard, S. A., Squires, K. C., Bauer, J. W., \& Lindsay, P. H. (1971). Evoked potential correlates of auditory signal detection. Science, 172, 1357-1360.

Howarth, C. I., \& TREISMAN, M. (1958). Lowering of an auditory threshold produced by a light signal occurring after the threshold stimulus. Nature, 182, 1093-1094.

J ACOBSON, E. (1911). Experiments on the inhibition of sensations. Psychology Review, 18, 24-53.

KaRLOVICH, R. S. (1969). Auditory thresholds during stroboscopic visual stimulation. Journal of the Acoustical Society of America, 45, $1470-1473$.

KarLoviCh, R. S., \& Moore, E. J. (1971). Stability of narrow-band noise thresholds during colored visual stimulation. Journal of Auditory Research, 11, 73-78.

Klayman, B. (1973). Detection and the timing of a hetermodal cue: Auditory-visual interaction. Journal of Psychology, 83, 201-214.

MARKov, P. O. (1960). The problem of the influence of extremely intense optic stimulation on the visual, auditory, and cutaneous analyzers in man. Biofizika, 5, 677-684.

OAtman, L. C. (1971). Role of visual attention on auditory evoked potentials in unanesthetized cats. Experimental Neurology, 32, 341-356.
OATMAN, L. C. (1976). Effects of visual attention on the intensity of auditory evoked potentials. Experimental Neurology, 51, 41-53.

OATMAN, L. C. (1982). Spectral analysis of cortical EEG activity during visual attention. Physiological Psychology, 10, 336-342.

OAtman, L. C., \& ANderson, B. W. (1977). Effects of visual attention on tone burst evoked auditory potentials. Experimental Neurology, 57, 200-211.

Oatman, L. C., \& ANderson, B. W. (1980). Suppression of the auditory frequency following response during visual attention. Electroencephalography and Clinical Neurophysiology, 49, 314-322.

O'HARE, J. J. (1956). Intersensory effect of visual stimuli on the minimum audible threshold. Journal of General Psychology, 54, 167-170.

Paul, D. D., \& SuTton, S. (1972). Evoked potential correlates of response criterion in auditory signal detection. Science, 177, 362-364.

SeIf, T. F., \& Howard, J. H. (1975). Stimulus compatibility effects of an accessory visual stimulus on auditory sensitivity. Perception \& Psychophysics, 17, 504-510.

Sheridan, J. A., Cimbalo, R. S., Sills, J. A., \& Alluisi, E. A. (1966). Effects of darkness, constant illumination, and synchronized photic stimulation on auditory sensitivity to pulsed tones. Psychonomic Science, 5, 311-312.

Shigehisa, T., \& Symons, J. R. (1973a). Effect of intensity of visual stimulation on auditory sensitivity in relation to personality. British Journal of Psychology, 64, 205-213.

Shigenisa, T., \& SYmons, J. R. (1973b). Reliability of auditory responses under increasing intensity of visual stimulation in relation to personality. British Journal of Psychology, 64, 375-381.

SIEGEL, J. (1968). A rapid procedure for locating deep electrode placements. Physiology \& Behavior, 3, 203-204.

SNIDER, R., \& NIEMER, W. T. (1961). A stereotaxic atlas of the cat brain. Chicago: University of Chicago Press.

TAYLOR, R. L. (1974). An analysis of sensory interaction. Neuropsychologia, 12, 65-71.

THOMPSON, R. F., Voss, J. F., \& BRogden, W. J. (1958). Effect of brightness of simultaneous visual stimulation on absolute auditory sensitivity. Jourmal of Experimental Psychology, 55, 45-50.

WATkINs, W. H. (1966). Photic facilitation of tonal signal detection in a forced-choice situation. Psychonomic Science, 6, 477-478.

(Manuscript received April 16, 1984; revision accepted for publication June 18, 1984.) 